\title{
Deploying Road Side Units in Sparse Vehicular Networks: What Really Works and What Does Not
}

\author{
Andre B. Reis, Susana Sargento, Filipe Neves, and Ozan K. Tonguz
}

\begin{abstract}
The 802.11p/WAVE standard relies on the presence of Onboard Units (OBUs) and Roadside Units (RSUs) for communications in vehicular networks. In this paper, we study the benefits of deploying RSUs to improve communications in highway scenarios. We develop an analytical model to analyze communication delay in a highway scenario with bidirectional traffic, considering both connected and disconnected RSUs, and validate our model via simulations and experimental measurements with 802.11p equipment. Contrary to conventional wisdom, our results show that significant benefits of RSUs in terms of connectivity and message dissemination can only be achieved when the deployed RSUs are interconnected. Conversely, deploying a large number of disconnected RSUs will lead to little or no benefit in message dissemination delay.
\end{abstract}

\section{INTRODUCTION}

$\mathbf{T}$ HE communication between vehicles in sparse vehicular ad hoc networks can be characterized by very high transmission delays, which adversely affect the quality of the communications. These delays pose a significant problem for safety messages, which should reach nearby vehicles as quickly as possible when an accident occurs, or when vehicle and road safety information need to be disseminated.

The packet delivery delay experienced in end-to-end communication scenarios between disconnected vehicles has been modeled and analyzed in previous works (see, for example, [1]). This delivery delay is known as the re-healing time. It was shown that this time can be larger than 100 seconds in multi-hop disconnected communication scenarios, which is detrimental for vehicular communications.

Such results and findings provide motivation for the deployment of Road Side Units (RSUs) to improve communication between vehicles on a highway. These infrastructure nodes are fixed base stations deployed along the road with the goal of increasing the overall coverage of a vehicular network. They can be equipped with better hardware than the units used in the vehicles, and can have less power and cost constraints. When used as fixed points for communication on highways, they are expected to enhance the network's performance and improve

Andre Reis and Ozan Tonguz are with Carnegie Mellon University, ECE Dept., Pittsburgh, PA 15213-3890, USA (e-mail: abreis@cmu.edu, tonguz@ece.cmu.edu). Andre Reis, Filipe Neves, and Susana Sargento are with Universidade de Aveiro, Instituto de Telecomunicacoes, 3810-193 Aveiro, Portugal (email: \{abreis,f.neves,susana\}@ua.pt).

Support for this research was provided in part by the T-SET University Transportation Center sponsored by the US Department of Transportation under Grant No. DTRT12-G-UTC11; by a CMU-Portugal ICTI Grant; by the Fundacao para a Ciencia e a Tecnologia (Portuguese Foundation for Science and Technology) through the Carnegie Mellon Portugal Program, under Grant SFRH/BD /67145/2009; and by the Fundacao para a Ciencia e a Tecnologia under Project PEst-OE/EEI/LA0008/2013. the propagation delay of messages between the several disconnected vehicles. A network of RSUs can also connect to a backbone, enabling access to other Wide Area Networks (WANs) or to the Internet. Although the presence of these units may significantly improve communication performance, a careful study of the associated improvement needs to be conducted, since the cost of deploying and supporting RSUs in vehicular environments can be very high.

The existing studies on RSUs are mostly focused on vehicle to infrastructure (V2I) communications (for dissemination of information or Internet access) [2]-[21]. Unfortunately, most of these studies only consider unidirectional traffic or assume that the RSUs are connected to a backbone. Such studies therefore provide little insight into the quality of communications when one deploys stand-alone RSUs.

The aim of this paper is to provide a comprehensive study of the impact of RSUs on the quality of the communications in highway scenarios, considering bi-directional traffic, where cars in one direction can relay traffic to the cars in the opposite direction. We present a detailed study based on analysis, simulations, and an experimental testbed, which provides insight into the tradeoff between network packet delay and number of RSUs deployed.

To this end, we develop mathematical models to determine the average delay of a packet between a disconnected source-destination pair in the presence of RSUs as relays or broadcasters of information. The models cover both the singlegap (the disconnection between adjacent clusters) and multigap communication scenarios. We study both the scenarios of disconnected RSUs, where RSUs are deployed without a physical connection between them, and interconnected RSUs, where RSUs are connected through fiber or wireless links.

Our analytical results are verified via extensive Monte Carlo simulations and further validated with empirical measurements, with real scenarios in the road, where cars and RSUs communicate through our own implementation of IEEE 802.11p/WAVE technology [22]. The results validate the accuracy of the proposed models, and highlight the disparity between deployment of disconnected and interconnected RSUs. For single-gap communications with disconnected $R S U$ support, the transmission delay can be reduced by $15 \%$ to $30 \%$; for traversing multiple gaps, a reduction of at most $25 \%$ in endto-end delay is possible. With connected RSUs the decrease in delay can be of several orders of magnitude, depending on the desired Region of Interest (RoI). These results are critical for the deployment of RSUs on highways: on the one hand, they show that the improvement by disconnected RSUs (where they are used as broadcasters of information) 
is quite modest even if the density of RSUs is very large; on the other hand, they show that connected RSUs (acting as relays of information) are the better choice for providing high quality communications and better connectivity in vehicular highway scenarios. Through this key result, we provide major insights into how this interconnected RSU scenario can be implemented on highways.

This work's key contributions can be summarized as follows:

- Developing an analytical framework for characterizing re-healing time in sparse highway networks, for two different types of RSU deployments (disconnected and interconnected), based on core traffic, network, and infrastructure deployment parameters.

- Showing, via the analytical models and Monte Carlo simulations, the substantial difference in performance between disconnected and interconnected RSUs. In addition to this key result, we also provide important insights into the influence that the density of RSUs and their radio range have in re-healing time.

- Confirming, through empirical measurements taken from a testbed that involved real Dedicated Short-Range Communication (DSRC) enabled vehicles and RSUs, that the predictions of the models in this work are accurate and realistic. There is an excellent match between the measurement results and the results predicted by our analytical models.

The remainder of this paper is organized as follows. Section II describes the vehicle and network traffic model, introducing the base analytical model. Section III describes the analytical models developed to model delays in disconnected and interconnected RSU deployments. The simulation and analytical results are depicted in Section IV, and the experimental results and testbed setup are given in Section V. A discussion is provided in Section VI, and related work is presented in Section VII. Finally, concluding remarks are given in Section VIII.

\section{Vehicle And Network Traffic Model}

\section{A. Parameters of Sparse Vehicular Networks}

An ad-hoc network operating on a highway with vehicles as network nodes can be fully characterized by a discrete set of parameters. Research in [1] presents an empirical study of traffic characteristics on the Interstate 80 highway in California, for various times of day, based on the analysis of extensive sets and traces of real freeway data, independently collected by the Berkeley Highway Laboratory (BHL) [23].

This study has shown that late-night traffic is tendentiously low volume and high speed. When a vehicular network is formed, the low density of vehicles under such conditions leads to network sparsity - i.e., a network that is prone to disconnected gaps between its nodes. This research is focused on methods to overcome such disconnection, and therefore, late-night and low volume traffic is the scenario that is most relevant to this work.

The inter-vehicle spacing of this type of traffic has been shown, in [1], to follow the well-known exponential distri- bution, which in turn allows one to derive various characteristics of the vehicular network that these vehicles create. The exponential distribution has been known to provide a reasonable representation of inter-vehicle spacing for lowdensity traffic [24].

It can therefore be seen that the critical parameters for such a network are: the vehicle traffic density $\lambda_{t}$, in vehicles entering one lane of the highway per unit of time; the vehicles' speed $v$, which, from empirical observation of late-night traffic, is seen to have a relatively low deviation from the mean, and can therefore be treated as a deterministic value; the number of vehicles per unit of distance, $\lambda_{s}=\lambda_{t} / v$; and on the network side, the expected radio range $R$ of each vehicle's DSRC radio. In this work, vehicles can travel in either the east-bound or the west-bound direction (see Figure 1).

From the exponential distribution of inter-cluster spacing, and the concept of a vehicle cluster (a group of vehicles traveling in the same direction, where each vehicle is in the radio range of at least one other vehicle), a series of equations that describe core characteristics of the sparse vehicular network can be derived. We now list the ones that are critical to our analysis. Figure 1 also illustrates some of these characteristics. For further details on this base traffic model and how each core characteristic is derived, please refer to [1].

The probability of being the last vehicle in a cluster is given by

$$
P_{d}=e^{-\lambda_{s} R}
$$

The Probability Distribution Function (PDF) of the spacing between cars in the same cluster, $S_{\text {intra }}$, as well as its expected value $E\left[S_{\text {intra }}\right]$, are given by

$$
\begin{aligned}
f_{S_{\text {intra }}}\left(s_{\text {intra }}\right) & =\frac{\lambda_{s} e^{-\lambda_{s} s_{\text {intra }}}}{1-e^{-\lambda_{s} R}} \\
E\left[S_{\text {intra }}\right] & =\frac{1}{\lambda_{s}}-\frac{R e^{-\lambda_{s} R}}{1-e^{-\lambda_{s} R}}
\end{aligned}
$$

Similarly, the distribution and expectation of the spacing between clusters (i.e., the gaps), $S_{\text {inter }}$, is given by

$$
\begin{aligned}
f_{S_{\text {inter }}}\left(s_{\text {inter }}\right) & =\lambda_{s} e^{-\lambda_{s}\left(s_{\text {inter }}-R\right)} \\
E\left[S_{\text {inter }}\right] & =R+\frac{1}{\lambda_{s}}
\end{aligned}
$$

The Probability Mass Function (PMF) of cluster size, $C_{N}$, in number of vehicles, is

$$
f_{C_{N}}\left(c_{n}\right)=P_{d}\left(1-P_{d}\right)^{c_{n}-1},
$$

and the mean cluster size is given by

$$
E\left[C_{N}\right]=\frac{1}{P_{d}}
$$

The vehicle cluster length distribution $C_{L}$, in meters, involves a more complex derivation. We refer to [26] where we provide a numerical calculation of the PDF of cluster length, $f_{C_{L}}\left(c_{l}\right)$. The expected cluster length is given by

$$
E\left[C_{L}\right]=\left(\frac{1}{P_{d}}-1\right)\left(\frac{1}{\lambda_{s}}-\frac{R e^{-\lambda_{s} R}}{1-e^{-\lambda_{s} R}}\right)
$$

With the deployment of fixed infrastructure units in the vehicular network, two new parameters are introduced: the 


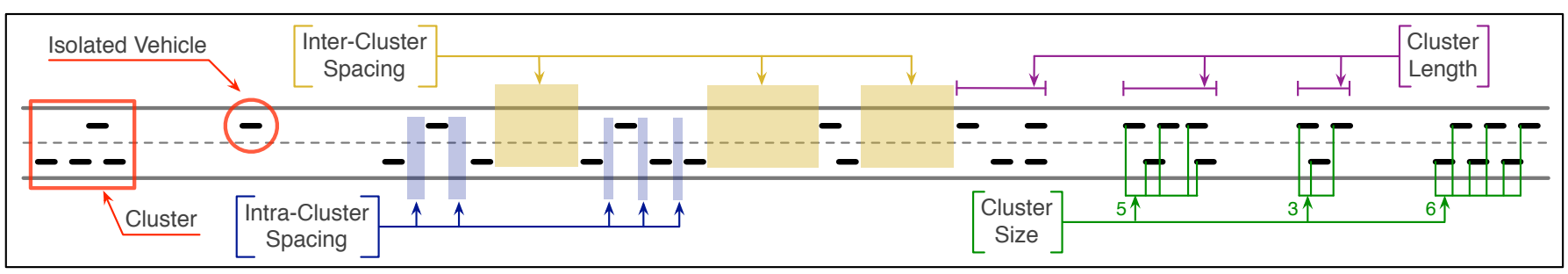

Figure 1. A standard highway scenario depicting several characteristics of sparse vehicular networks.

distance $C_{I}$, in meters, between each RSU and the next, and the radio range $R_{I}$ of these units, also in meters. Being specialized equipments, we observe that an RSU's physical hardware can be improved from the one deployed in vehicles, to enable increased transmission power and/or antenna gain, which in turn would result in an improved communication range.

\section{B. Analytical Modeling of Sparse Vehicular Networks}

The transmission scenario that will be under consideration for the remainder of the paper is the transmission of a message from a source vehicle, denoted as $S r c$, to a destination vehicle, $D s t$, in a two-lane, bidirectional road. To better characterize each lane's traffic, the vehicle density is denoted as $\lambda_{e}$ and $\lambda_{w}$, and the vehicle's speed as $v_{e}$ and $v_{w}$, depending on whether we refer to east-bound or west-bound traffic.

$S r c$ and Dst in our scenario must necessarily be members of two separate clusters, with any number of intermediate clusters in between - their exact number depends on the distance between $S r c$ and Dst and on network and traffic parameters. Therefore, there is at least one communication gap between Src and Dst that cannot be overcome without the aid of a third entity.

This entity is an opposite-lane vehicle, denoted as $Z$, that receives the message from $S r c$, stores it, and delivers it to Dst once it communicates with Dst. This mechanism is called Store-Carry-Forward transmission, and the time for the message to be transmitted across a region of disconnection is designated the Re-Healing Time.

For a message transmission requiring one or more gaps to be traversed, the need for 're-healing' happens when the last car in a cluster (tail) has received a message and is unable to relay the message to the head of the following cluster. Using the last vehicle as a point of reference, which we consider to be the $S r c$ vehicle from now on, two main scenarios can be identified:

- The best-case scenario, which occurs when the Src vehicle is in the range of an opposite-lane vehicle capable of receiving and relaying the message (Figure $2 \mathrm{a}$ );

- The worst-case scenario, which occurs when there is no opposite-lane vehicle in range of Src, and thus Src must wait for one such vehicle to relay the message (Figure 2b).

These scenarios are fundamental for the understanding of how messages propagate in sparse vehicular networks, and are an important tool for understanding the contribution of infrastructure deployments. We now briefly describe the two scenarios.

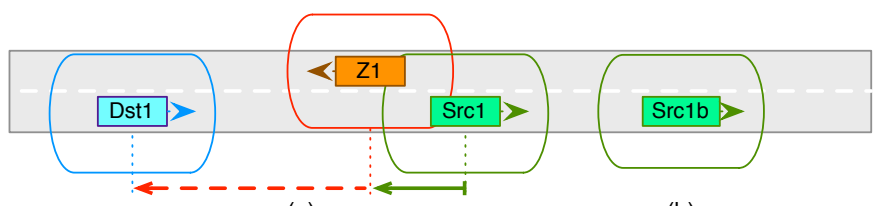

(a)

(b)

Figure 2. Examples of a) a best-case scenario; b) a worst-case scenario.

1) Best-Case Scenario: In a best-case scenario, depicted in Figure 2a, the source vehicle $S r c l$ is directly connected to vehicle $Z$ on the opposite lane at the time it receives a message for Dst1. This scenario then considers two possibilities:

- No vehicles in Z's cluster are in range of Dst1, in which case the head vehicle of Z's cluster must wait until it moves in range of Dstl; this scenario occurs with probability $p_{10}$ and its re-healing time is denoted as $E\left[T_{r_{10}}\right]$.

- Vehicle $Z$ or a vehicle in its cluster might be directly connected to Dst1, in which case the re-healing time is instantaneous; this scenario occurs with probability $(1-$ $\left.p_{10}\right)$, and its re-healing time is denoted as $E\left[T_{r_{11}}\right]$.

Given these two events, it can be shown that the mean rehealing time in a best-case scenario is given by

$$
\begin{aligned}
& E\left[T_{r_{1}}\right]=E\left[T_{r_{10}}\right] \cdot p_{10}+E\left[T_{r_{11}}\right] \cdot\left(1-p_{10}\right) \\
= & \left\{\left(1-P_{d}\right) \cdot \frac{1}{v_{e}+v_{w}} \cdot\left\{\frac{1}{\lambda_{e}}-\frac{1}{2} E\left[S_{\text {intra }}\right] E\left[C_{N} \mid C_{N} \leq k\right]\right\}\right. \\
& \left.+P_{d} \cdot \frac{1}{v_{e}+v_{w}} \cdot\left(R+E\left[S_{\text {inter }}\right]-R\right)\right\} \cdot p_{10}
\end{aligned}
$$

2) Worst-Case Scenario: In a worst-case scenario, as depicted in Figure 2b, the disconnected vehicle $\mathrm{Srclb}$ does not have any opposite-lane vehicle in its range. Therefore, for the message to be carried across the gap, it must now wait for an opposite-lane vehicle to move into its range, and then for that vehicle to carry the message to Dst1. Two delay components are discernible: the temporal delay from $\operatorname{Src}$ to $Z$ (denoted as $E\left[T_{r_{20}}\right]$ ), and then from $Z$ to $D s t$ (denoted as $E\left[T_{r_{21}}\right]$ ).

The mean re-healing time in a worst-case scenario can be shown to be

$$
\begin{aligned}
& E\left[T_{r_{2}}\right]=E\left[T_{r_{20}}\right]+E\left[T_{r_{21}}\right] \\
& =p_{20}^{1} \cdot \frac{E\left[C_{L}\right]}{v_{e}+v_{w}}+\left(1-p_{20}^{1}\right) \cdot \frac{1}{2 \lambda_{w}\left(v_{e}+v_{w}\right)}+\frac{E\left[S_{\text {inter }}\right]}{v_{e}+v_{w}},
\end{aligned}
$$


where $p_{20}^{1}=P\left[C_{L}<E\left[S_{\text {inter }}^{\prime}\right]-2 R\right]$, is the probability that $S r c$ is in a small cluster. Details on the distinction between small and large clusters and on how this result is reached are outlined in [1].

3) Mean Per-Gap Re-healing Time: The worst-case scenario occurs with probability $P_{d}^{\prime}=e^{-2 R \lambda_{w}}$, the probability of having no opposite-lane vehicles in a range of $2 R$, which follows from the exponential distribution of vehicle spacing. It also follows that the best-case scenario occurs with probability $1-P_{d}^{\prime}$, and thus the mean re-healing time per gap is given by

$$
E\left[T_{r}\right]=\left(1-P_{d}^{\prime}\right) \cdot E\left[T_{r_{1}}\right]+P_{d}^{\prime} \cdot E\left[T_{r_{2}}\right] .
$$

4) Multi-Gap Delay: Often, a safety message will need to overcome many gaps before it reaches its destination or is delivered to all vehicles in a predetermined area of interest. As a result of the memoryless property of the exponential distribution [25], all gaps on the road are statistically independent, and can be analyzed individually.

In order to determine the mean re-healing time required for a message to be delivered to a given point in the road, one must first determine how many gaps exist between the source and the destination vehicles. Given the distance $d$ between both vehicles, computing the mean number of gaps, $G_{C}$, from the cluster length and intercluster spacing, is straightforward:

$$
G_{C}(d)=\frac{d}{E\left[C_{L}\right]+E\left[S_{\text {inter }}\right]}
$$

The mean re-healing time involving multiple gaps can be determined by multiplying the Gap Count $G_{C}$ with the pergap re-healing time, $E\left[T_{r}\right]$.

\section{COMMUNICATION MODEL FOR INFRASTRUCTURE-SUPPORTED VEHICULAR NETWORKS}

This section presents the analytical models to characterize vehicular networks with disconnected and connected RSU deployments. Together with the simulation data and the empirical studies, these constitute the core research that is presented in this paper. One can envision several specific scenarios where RSUs can provide significant benefits to communications:

- RSUs as communication relays;

- RSUs as broadcasters of information (one-time or repeated information);

- RSUs as infrastructure communication points to and from a WAN (e.g., Internet).

When one considers the benefit of having RSUs assist communications in sparsely connected networks, the most critical scenario is the first one, where we envision deployments of RSUs to enable relaying of information when there is severe disconnection between vehicles. Therefore, this paper specifically addresses this scenario, where we consider that vehicles flow in both directions and that RSUs, if present, are helping to relay information between disconnected sources and destinations.
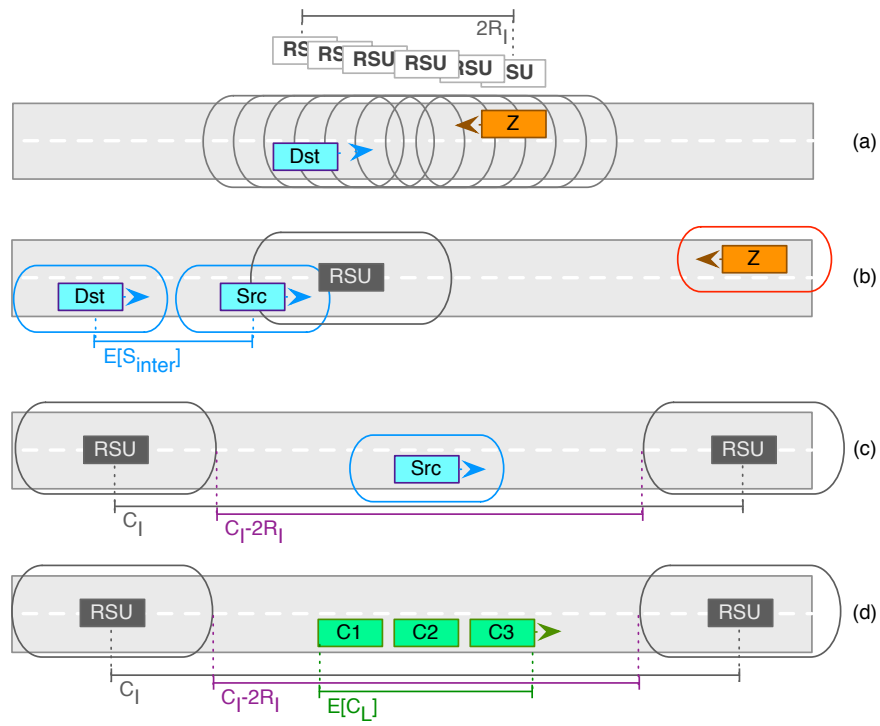

Figure 3. (a) Favorable positions for RSUs in reducing spatial distance between $S r c$ and $Z$; (b) a scenario where the RSU can store and forward a message from $S r c$ to $D s t$ faster than $Z$ would; (c), (d) example scenarios of an isolated vehicle, and of clustered vehicles, respectively.

\section{A. Disconnected RSU Scenarios}

The most straightforward scenario is a pure deployment of RSUs along the road, regularly spaced at a fixed distance, with no backbone to interconnect the RSUs. Our first model characterizes re-healing time on a highway where RSUs are deployed in a stand-alone or disconnected manner.

Working with the analytical framework described in Section II-B, we determine which communication scenarios can benefit from the presence of RSUs. Best-case and worstcase scenarios are evaluated independently, as each lead to a different set of benefits.

1) Best-Case Scenario - RSU Reduces Spatial Distance: In a best-case scenario, as previously shown in Figure 2a, where the source vehicle $S r c$ is directly connected to a vehicle $Z$ in the opposite lane to carry its message, improvements are observed when an RSU is positioned in a way where it can forward the message from $Z$ to the destination $D s t$, effectively acting as a bridge between the two vehicles.

To obtain the average reduction in the distance that $Z$ needs to travel, we first note that, if the RSUs are placed at fixed intervals of $C_{I}$ meters, then the probability of finding an RSU at an exact point in space is a uniformly distributed random variable in $\left[0, C_{I}\right]$ :

$$
f_{R S U}(r)= \begin{cases}1 / C_{I} & 0<r<C_{I} \\ 0 & \text { otherwise }\end{cases}
$$

We now refer to Figure 3a. By taking as reference the point in time where the vehicles $D s t$ and $Z$ close in and reach a distance of $2 R_{I}$ of one another, we see a range of positions where an RSU can be found that would allow for the RSU to act as a relay between the two vehicles. In this scenario, if no RSUs were present, the two vehicles would have to close in to a distance of $R$ to be able to communicate, i.e., they would have to travel a distance of $2 R_{I}-R$.

The range of favorable RSU positions is between: 
- The RSU being right on top of either vehicle Dst or Z. This is the least favorable scenario, and results in no improvement in delay.

- The RSU being in front of vehicle $D s t$ by $R_{I}$. This is the most favorable scenario, where the travel distance reduction is highest (by $2 R_{I}-R$ meters) and vehicles can communicate immediately.

From the last point, we observe that the reduction in travel distance increases linearly from $x=0$ to $R_{I}$ and decreases linearly from $R_{I}$ to $2 R_{I}$, with $x$ as the distance from the RSU to Dst. The travel distance reduction, $G(x)$, is given by

$$
G(x)= \begin{cases}\left(2-\frac{R}{R_{I}}\right) x & 0 \leq x<R_{I} \\ -\left(2-\frac{R}{R_{I}}\right) x+2\left(2 R_{I}-R\right) & R_{I} \leq x<2 R_{I}\end{cases}
$$

Lemma 1. The mean reduction in travel distance achieved by the presence of an RSU in a favorable position to relay messages is given by

$$
E\left[L_{I}^{1}\right]=\int_{0}^{2 R_{I}} G(x) f_{R S U}(x) \mathrm{d} x=\frac{\left(2 R_{I}-R\right)\left(R_{I}^{2}+R^{2}\right)}{2 R_{I} C_{I}} .
$$

Proof of Lemma 1: Follows from eqs. (13) and (14). The range of favorable RSU locations is $\left[0, R_{I}\right]$, the probability of having an RSU in such location is given by $f_{R S U}$, and each point in this range represents a reduction in travel length given by $G(x)$.

In a multi-gap scenario, an improvement is only seen if a vehicle in the new cluster has a new opposite-lane vehicle in range to act as a relay. Refer to Figure 3a: if vehicle Dst cannot immediately forward the message to a new oppositelane vehicle, then it will have to use vehicle $Z$ again as the new relay (which would then be the closest opposite-lane vehicle), thus nullifying possible gains.

Lemma 2. The probability of a cluster of cars having at least one opposite-lane vehicle in range is given by

$$
\begin{aligned}
P_{d_{C}} & =1-\operatorname{Pr}\left[\text { no opposite-lane vehicles in a range of } C_{L}\right] \\
& =1-e^{-\lambda_{w} \cdot E\left[C_{L}\right]}
\end{aligned}
$$

Proof of Lemma 2: Due to the exponential characteristic of traffic in both lanes, the probability of having no vehicles in a given range $L$ will be given by $e^{-\lambda L}$ (follows from zero realizations in a homogenous Poisson Point Process of rate $\lambda$ ). The desired probability is the complement of having zero vehicles in the range of interest, which corresponds to the length of a cluster. Refer to [26] for the definition of $E\left[C_{L}\right]$.

Lemma 3. The mean reduction in travel distance achieved by a regularly spaced deployment of disconnected RSUs is given by

$$
\begin{aligned}
E\left[L_{I}\right] & =E\left[L_{I}^{1}\right] \cdot P_{d_{C}} \\
& =\frac{\left(2 R_{I}-R\right)\left(R_{I}^{2}+R^{2}\right)}{2 R_{I} C_{I}}\left(1-e^{-\lambda_{w} \cdot E\left[C_{L}\right]}\right)
\end{aligned}
$$

Proof of Lemma 3: Follows from Lemmas 1 and 2.
The new best-case re-healing time, $E\left[T_{r_{1}}^{\prime}\right]$, is the result of subtracting $E\left[L_{I}\right]$ (from Lemma 3 ) to the distance components in eq. (9):

$$
\begin{aligned}
E\left[T_{r_{1}}^{\prime}\right]= & \left\{\frac { ( 1 - P _ { d } ) } { v _ { e } + v _ { w } } \cdot \left(\frac{1}{\lambda_{e}}-\frac{1}{2} E\left[S_{\text {intra }}\right] E\left[C_{N} \mid C_{N} \leq k\right]\right.\right. \\
& \left.\left.-E\left[L_{I}\right]\right)+\frac{P_{d}}{v_{e}+v_{w}}\left(E\left[S_{\text {inter }}\right]-E\left[L_{I}\right]\right)\right\} \cdot p_{10}
\end{aligned}
$$

2) Worst-Case Scenario - RSU as Message Relay: In a worst-case scenario, the source vehicle is not connected to any vehicle capable of forwarding its message, and must wait for one to come into range.

With the presence of RSUs, a new scenario where an RSU acts as a relay becomes possible. This is shown in Figure $3 b$. This occurs when the delay to forward a message through an opposite-lane vehicle is larger than the delay for the source to get a message to an RSU, plus the delay for the destination to reach that RSU.

Lemma 4. The probability of having an RSU act as a message relay between two disconnected clusters (in a shorter time than an opposite-lane vehicle) is given by

$$
p_{2 A}=e^{\lambda_{w}(R-K)}
$$

with $K=2\left[\left(E\left[S_{\text {inter }}\right]-R_{I}+C_{I} / 2\right)\left(v_{e}+v_{w}\right)-\left(E\left[S_{\text {inter }}\right]-\right.\right.$ $R)] / v_{e}$.

Proof of Lemma 4: We begin by determining an expression for the probability of having the RSU as the main relay. This event occurs when the time it takes for $S r c$ to find a vehicle $Z$, and then for $Z$ to reach $D s t$, is larger than the time it would take for $S r c$ to reach an RSU, and for that RSU to come in range of Dst:

$$
P\left[\frac{S_{\text {inter }}-R+S_{\text {inter }}^{\prime} / 2}{v_{e}+v_{w}}>\frac{S_{\text {inter }}-R_{I}+C_{I} / 2}{v_{e}}\right],
$$

where $S_{\text {inter }}^{\prime}$ is the inter-cluster spacing for vehicles in the opposite-lane. Rearranging the inequality as a function of $S_{\text {inter }}^{\prime}$, we obtain a probability in the form of $P\left[S_{\text {inter }}^{\prime}>K\right]$, with $K=2\left[\left(E\left[S_{\text {inter }}\right]-R_{I}+C_{I} / 2\right)\left(v_{e}+v_{w}\right)-\left(E\left[S_{\text {inter }}\right]-\right.\right.$ $R)] / v_{e}$.

$p_{2 A}$ can now be determined by the inverse Cumulative Distribution Function (CDF) of $S_{\text {inter }}^{\prime}$ :

$$
\begin{aligned}
p_{2 A} & =1-P\left[S_{\text {inter }}^{\prime}<K\right] \\
& =1-\int_{R}^{K} \lambda_{w} \cdot e^{-\lambda_{w}\left(S_{\text {inter }}^{\prime}-R\right)} \cdot \mathrm{d} S_{\text {inter }}^{\prime} \\
& =e^{\lambda_{w}(R-K)}
\end{aligned}
$$

Lemma 5. The average re-healing time when an RSU is acting as the main relay is given by

$$
E\left[T_{r_{2 A}}\right]=\left(\frac{C_{I}-2 R_{I}}{2}+R+\frac{1}{\lambda_{e}}\right) \cdot \frac{1}{v_{e}}
$$


Proof of Lemma 5: The re-healing time for the scenario addressed in Lemma 4 is given by the average distance from Src to the RSU, plus the distance from Src to Dst, which corresponds to the inter-cluster spacing in the lane of interest. Since a vehicle can be, at most, $C_{I}-2 R_{I}$ meters away from an RSU, the average distance to an RSU is $\left(C_{I}-2 R_{I}\right) / 2$ :

$$
E\left[T_{r_{2 A}}\right]=\left(\frac{C_{I}-2 R_{I}}{2}+E\left[S_{\text {inter }}\right]\right) \cdot \frac{1}{v_{e}}
$$

and with eq. (5), Lemma 5 follows.

3) Worst-Case Scenario - RSU Reduces Spatial Distance: If the scenario described in Section III-A2 does not occur, the standard worst-case scenario applies. Re-healing time is the sum of two components: Src must wait until an opposite-lane vehicle $Z$ comes into range, and $Z$ must carry this message to Dst. For the worst-case scenario, we observe that an RSU brings no advantage to the temporal delay between $\operatorname{Src}$ and $Z$. Even if the message from $S r c$ were to reach $Z$ with the help of an RSU, Z's time to deliver the message to Dst would not change.

The benefit of an infrastructure unit, in this scenario, comes as a reduction in the distance that $Z$ needs to travel to reach $D s t$ - this reduction is the same as the one shown in Lemma 3.

Lemma 6. In a worst-case scenario with RSU support, the re-healing time from $\mathrm{Z}$ to $\mathrm{Dst}$ is given by

$$
E\left[T_{r_{21}}\right]=\left(R+\frac{1}{\lambda_{e}}-E\left[L_{I}\right]\right) \cdot \frac{1}{v_{e}+v_{w}}
$$

Proof of Lemma 6: Follows from Lemma 3 and eq. (10).

Due to the inclusion of the new scenario as described in Section III-A2, the worst-case total re-healing time is now simply

$$
E\left[T_{r_{2}}^{\prime}\right]=E\left[T_{r_{2 A}}\right] \cdot p_{2 A}+E\left[T_{r_{2 B}}\right] \cdot\left(1-p_{2 A}\right)
$$

where $E\left[T_{r_{2 B}}\right]$ is the previous worst-case re-healing time, from eq. (10), after replacing $E\left[T_{r_{21}}\right]$ with Lemma 6 .

With the best-case and worst-case scenarios appropriately extended for the presence of RSUs, we reach the global pergap re-healing time equation, which can be stated by the following theorem.

Theorem 1. The re-healing time for a network with disconnected RSU support is given by

$$
\begin{aligned}
& E\left[T_{r}^{\prime}\right]=\left(1-P_{d}^{\prime}\right) \cdot E\left[T_{r_{1}}^{\prime}\right]+P_{d}^{\prime} \cdot E\left[T_{r_{2}}^{\prime}\right] \\
& =\left(1-e^{-\lambda_{w} 2 R}\right) \cdot\left\{\frac { ( 1 - P _ { d } ) } { v _ { e } + v _ { w } } \cdot \left[\frac{1}{\lambda_{e}}-\frac{1}{2} E\left[S_{\text {intra }}\right]\right.\right. \\
& \left.\left.\times E\left[C_{N} \mid C_{N} \leq k\right]-E\left[L_{I}\right]\right]+\frac{P_{d}}{v_{e}+v_{w}}\left(E\left[S_{\text {inter }}\right]-E\left[L_{I}\right]\right)\right\} \\
& \times p_{10}+e^{-\lambda_{w} 2 R} \cdot\left\{e^{\lambda_{w}(R-K)}\left(\frac{C_{I}-2 R_{I}}{2}+R+\frac{1}{\lambda_{e}}\right) \cdot \frac{1}{v_{e}}\right. \\
& +\left(1-e^{\lambda_{w}(R-K)}\right)\left[p_{20}^{1} \cdot \frac{E\left[C_{L}\right]}{v_{e}+v_{w}}+\left(1-p_{20}^{1}\right) \cdot \frac{1}{2 \lambda_{w}\left(v_{e}+v_{w}\right)}\right. \\
& \left.\left.+\left(R+\frac{1}{\lambda_{e}}-E\left[L_{I}\right]\right) \cdot \frac{1}{v_{e}+v_{w}}\right]\right\}
\end{aligned}
$$

Proof of Theorem 1: Follows from eqs. (11), (18) and (25), and Lemmas 1-6.

To conclude the analysis on disconnected RSU support, we recall Section II-B4 to note that the multi-gap rehealing time with disconnected RSUs can be computed by following the same rationale as before. The introduction of RSUs in the road does not change the number or the length of the clusters, so the average number of gaps in a given span remains the same.

\section{B. Interconnected RSU Scenarios}

In a highway where road-side units are deployed and interconnected, the network of RSUs is capable of quickly forwarding traffic over large distances by relaying traffic through its interconnected backbone. To characterize message propagation delay in this scenario, we break away from the previous models and take a more pragmatic approach where the message will be preferentially relayed through the connected network of RSUs. This approach is reasonable given the fact that we are primarily interested in characterizing re-healing time for messages that need to traverse multiple disconnected clusters over medium to high spans of road, and thus relying on the interconnected RSUs will always be the preferred method.

Definition 1. In an interconnected $R S U$ scenario, if the source and destination vehicles are spaced sufficiently far away (more than the RSU spacing), messages are primarily relayed through RSUs. Therefore, a vehicle is considered disconnected if it is not in range of an RSU. With interconnected RSUs, rehealing time is the average time a disconnected vehicle waits until it can contact an RSU, either by itself (single-hop) or through other vehicles in its cluster (multi-hop).

The total re-healing time is both the average delay for the Src vehicle to reach an RSU and the average delay for the Dst vehicle to also reach an RSU. These events are indistinguishable, as the goal of both vehicles is to establish contact with an RSU.

Two main cases are identifiable:

- Isolated vehicle: if the vehicle is isolated, its re-healing time is the time for the vehicle to contact an RSU.

- Clustered vehicle: if the vehicle is part of a cluster, its re-healing time is the shortest time for any vehicle in the cluster to reach an RSU - intra-cluster communication can relay the message to the RSU as necessary.

We now derive analytical models that characterize the rehealing time in each of these main cases.

1) Isolated Vehicles: For an isolated vehicle, we identify the following three metrics: probability that an isolated vehicle is disconnected from an RSU, $\operatorname{Pr}\left[V_{d}\right]$; average delay for a disconnected vehicle to reach an RSU's radio range, $E\left[T_{r_{V}} \mid V_{d}\right]$; and probability that a vehicle is isolated, $\operatorname{Pr}\left[C_{N}=1\right]$ (i.e., the vehicle is not part of a cluster). This scenario is depicted in Figure 3c.

The probability that an isolated vehicle is disconnected from an RSU at the time of transmission or reception of a message is obtained in a straightforward manner from the density of 
RSUs and their radio range. For an RSU separation distance of $C_{I}$, and an RSU radio range of $R_{I}$, the proportion of road not covered by the RSUs' radio ranges equals the probability that, at any given point in time, a vehicle is disconnected from its nearby RSUs:

$$
\operatorname{Pr}\left[V_{d}\right]=\frac{C_{I}-2 R_{I}}{C_{I}}=1-\frac{2 R_{I}}{C_{I}}
$$

If the vehicle is disconnected, then it must be located in the span of road between two consecutive RSUs' radio ranges, i.e., in Figure $3 \mathrm{c}$ it must be located in the area with a length of $C_{I}-$ $2 R_{I}$. Statistically, it is safe to assume that, on average, the vehicle will be located in the center of this region; therefore, the average re-healing time is given by the time to traverse half the length of the region with no RSU coverage:

$$
E\left[T_{r_{V}} \mid V_{d}\right]=\frac{C_{I}-2 R_{I}}{2} \cdot \frac{1}{v_{e}}
$$

Lemma 7. In a highway with connected RSUs, the average re-healing time of an isolated vehicle is given by

$$
\begin{aligned}
E\left[T_{r_{V}}\right] & =E\left[T_{r_{V}} \mid V_{d}\right] \cdot \operatorname{Pr}\left[V_{d}\right]+E\left[T_{r_{V}} \mid \neg V_{d}\right] \cdot \operatorname{Pr}\left[\neg V_{d}\right] \\
& =\frac{C_{I}-2 R_{I}}{2 v_{e}} \cdot\left(1-\frac{2 R_{I}}{C_{I}}\right)
\end{aligned}
$$

Proof of Lemma 7: Follows from eqs. (27) and (28); the re-healing time associated with event $\neg V_{d}$ is zero, as the vehicle is connected.

2) Clustered Vehicles: In a cluster of vehicles, it is sufficient to have a single vehicle in the cluster in range of an RSU for all vehicles in the cluster to be able to communicate with the RSU. This requires vehicle-to-vehicle multi-hop communications in the cluster. The following definition is based on this observation:

Definition 2. In a highway where RSUs are deployed uniformly at a fixed distance of $C_{I}$, and where each $R S U$ has a radio range $R_{I}$, if the length of a cluster of vehicles is equal to or larger than $C_{I}-2 R_{I}$, then at least one vehicle in that cluster will always be directly connected to an RSU, and therefore all vehicles in the cluster are considered 'connected'.

For clustered vehicles, we observe that the following must occur for the re-healing time to not be zero:

- As per Definition 2, the length of the cluster the vehicle belongs to must be less than $C_{I}-2 R_{I}$. This event's probability is $P\left[C_{L}<C_{I}-2 R_{I}\right]$.

- If the cluster's length satisfies the above condition, it may still happen that one or more of the vehicles in the cluster are in range of an RSU in the time period where communication is requested. We denote the probability that this event does not occur by $\operatorname{Pr}\left[C_{d}\right]$.

Lemma 8. The probability that the length of a cluster is inferior to $C_{I}-2 R_{I}$ is given by

$$
P\left[C_{L}<C_{I}-2 R_{I}\right]=1-\frac{e^{-\frac{C_{I}-2 R_{I}}{\mu}}}{k R+e^{-\frac{R}{\mu}}}
$$

Proof of Lemma 8: See [26], where regression variables $k$ and $\mu$ are also defined.
Lemma 9. The expected length of a cluster, conditioned on $C_{L}<C_{I}-2 R_{I}$, is given by

$$
\begin{aligned}
& E\left[C_{L} \mid C_{L}<C_{I}-2 R_{I}\right]= \\
& \frac{e^{\frac{R+C_{I}}{\mu}} k R^{2}+2 e^{\frac{C_{I}}{\mu}}(R+\mu)-2 e^{\frac{R+2 R_{I}}{\mu}}\left(\mu+C_{I}-2 R_{I}\right)}{2\left(e^{\frac{C_{I}}{\mu}}-e^{\frac{R+2 R_{I}}{\mu}}+e^{\frac{R+C_{I}}{\mu}} k R\right)}
\end{aligned}
$$

Proof of Lemma 9: See [26].

Consider the scenario in Figure $3 \mathrm{c}$. For the cluster to be disconnected, the edge vehicles of the cluster must be in the region $\left[R_{I}, C_{I}-R_{I}\right]$. Therefore, by taking the center of the cluster as reference, it follows that the cluster's center must be in a region of length $\left(C_{I}-2 R_{I}\right)-E\left[C_{L} \mid C_{L}<C_{I}-2 R_{I}\right]$, otherwise one of the edge vehicles will be in range of an RSU. The cluster's center can be located anywhere in $\left[0, C_{I}\right]$. Therefore, the probability of a cluster being disconnected from the RSU network, $P\left[C_{d}\right]$, is given by

$$
\operatorname{Pr}\left[C_{d}\right]=\frac{\left(C_{I}-2 R_{I}\right)-E\left[C_{L} \mid C_{L}<C_{I}-2 R_{I}\right]}{C_{I}} .
$$

Let us consider Figure $3 \mathrm{c}$ again. Statistically speaking, it is correct to assume that the center of the cluster is in the middle of region $\left[0, C_{I}\right]$. Thus, the shortest re-healing time is the time for the frontmost vehicle in the cluster to reach the next RSU's radio range, which corresponds to a travel distance of $\left(C_{I}-2 R_{I}-E\left[C_{L} \mid C_{L}<C_{I}-2 R_{I}\right]\right) / 2$. The mean re-healing time for any vehicle in a cluster which is both disconnected and smaller than $C_{I}-2 R_{I}$ is given by

$$
\begin{aligned}
& E\left[T_{r_{C}} \mid C_{d} \cap C_{L}<C_{I}-2 R_{I}\right]= \\
& \quad \frac{\left(C_{I}-2 R_{I}\right)-E\left[C_{L} \mid C_{L}<C_{I}-2 R_{I}\right]}{2} \cdot \frac{1}{v}
\end{aligned}
$$

Lemma 10. The average re-healing time of a vehicle in a disconnected cluster is given by

$$
\begin{aligned}
& E\left[T_{r_{C}}\right]=E\left[T_{r_{C}} \mid C_{d}\right.\left.\cap C_{L}<C_{I}-2 R_{I}\right] \times \\
& \times \operatorname{Pr}\left[C_{d}\right] \cdot \operatorname{Pr}\left[C_{L}<C_{I}-2 R_{I}\right] \\
&=\frac{\left(C_{I}-2 R_{I}-E\left[C_{L} \mid C_{L}<C_{I}-2 R_{I}\right]\right)^{2}}{2 \cdot v_{e} \cdot C_{I}} \cdot\left(1-\frac{e^{-\frac{C_{I}-2 R_{I}}{\mu}}}{k R+e^{-\frac{R}{\mu}}}\right)
\end{aligned}
$$

Proof of Lemma 10: Follows from Lemmas 8 and 9, eqs. (32) and (33), and the fact that the specific events $\neg C_{d}$ and $C_{L} \geq C_{I}-2 R_{I}$ correspond to a re-healing time of zero.

3) Global Re-Healing Time Model: The final model for per-gap re-healing time, stated by the following theorem, is the consolidation of isolated vehicle and clustered vehicle scenarios.

Theorem 2. The per-gap re-healing time for a network with 
interconnected RSU support is given by

$$
\begin{aligned}
E\left[T_{r}\right]= & E\left[T_{r_{V}}\right] \cdot \operatorname{Pr}\left[C_{N}=1\right]+E\left[T_{r_{C}}\right] \cdot \operatorname{Pr}\left[C_{N}>1\right] \\
= & \frac{C_{I}-2 R_{I}}{2 v} \cdot\left(1-\frac{2 R_{I}}{C_{I}}\right) \cdot e^{-\lambda_{s} \cdot R} \\
& +\frac{\left(C_{I}-2 R_{I}-E\left[C_{L} \mid C_{L}<C_{I}-2 R_{I}\right]\right)^{2}}{2 v C_{I}} \\
& \times\left(1-\frac{e^{-\frac{C_{I}-2 R_{I}}{\mu}}}{k R+e^{-\frac{R}{\mu}}}\right) \cdot\left(1-e^{-\lambda_{s} \cdot R}\right)
\end{aligned}
$$

Proof of Theorem 2: Follows from Lemmas 7 and 10, and the Cluster Size PMF in eq. (6).

\section{EVAluation OF INFRASTRUCTURE-SUPPORTED NETWORKS}

In this section we present the results obtained with the analytical model proposed in Section III, and with a MonteCarlo simulation platform which is used for verifying the analytical model.

\section{A. Monte-Carlo Simulation Model}

Our custom-designed simulator, written in $\mathrm{C}++$, replicates a straight two-lane highway, with traffic flowing in both directions. Vehicles are generated at the beginning of each road at time intervals obtained from an exponential random number generator. Vehicles move at a constant speed, and there is no overtaking.

The transmission range of each vehicle and RSU can be controlled, and is set to $250 \mathrm{~m}$ unless otherwise stated. Radios are assumed to have perfect and instant communication, with no packet error when within the radio range, and no communication outside it. We also assume that, once a node receives a message, it immediately rebroadcasts it to every node in its vicinity. In Section V, we prove the validity of these assumptions for sparse networks with data from an experimental $802.11 \mathrm{p}$ testbed.

For each simulation, the setup is as follows: the simulator first runs for an arbitrarily long time, sufficient to fill the road on both lanes. Then, source and destination vehicles are picked in order to match the criteria of that particular simulation, e.g., if the goal is to transmit a packet between two vehicles which are $10 \mathrm{~km}$ apart, the simulator attempts to locate a pair of vehicles in the road that better matches that goal.

Once this process is completed, the source vehicle generates and broadcasts a single message. After the message is received by the destination vehicle, metrics are recorded and the simulation setup is discarded.

\section{B. Validation of Inner Model Characteristics}

1) Disconnected RSUs: The two main components of rehealing time in disconnected RSUs are the best-case and the worst-case re-healing time. For this analysis, we set up a $10 \mathrm{~km}$ road with RSUs deployed at $1 \mathrm{~km}$ intervals, and once both lanes were filled with traffic, a vehicle in the eastbound lane was randomly selected to broadcast a message. The simulator then measured the time for the message to reach the next cluster in the eastbound lane, and reported whether we were in a presence of a best-case or a worst-case scenario.

Figure $4 \mathrm{a}$ shows the analytical and simulation results for the two main components of the disconnected RSU model, for vehicle densities ranging from 213 to $745 \mathrm{veh} / \mathrm{h}$ (the same density is used in both directions). Figure $4 \mathrm{~b}$ shows the results of a simulation where $S r c$ and Dsc are $10 \mathrm{~km}$ apart, with disconnected RSUs deployed every $1 \mathrm{~km}$, and is thus a setup where both best case and worst case scenarios can occur randomly.

We observe a very good match between the predictions of our analytical model and the output of the simulations. Results show that, as the traffic density increases, the rehealing time of both best- and worst-case scenarios decreases, as expected. As a network grows sparser, so do the re-healing times; furthermore, the likelihood of being in the presence of a worst-case scenario also increases, which carries a higher penalty in re-healing time.

2) Interconnected RSUs: As explained in Section III-B, our approach for scenarios with interconnected RSUs considers that the RSUs are the primary entity relaying messages. Therefore, two main cases are considered: the case where the vehicle is isolated, and the case where it is part of a cluster.

A simulation is set up with a $10 \mathrm{~km}$ road, where RSUs are deployed at regular intervals and interconnected. We consider that, when an RSU receives a message, the message immediately becomes available at all other RSUs, which begin rebroadcasting that message. Figure $4 \mathrm{c}$ shows the re-healing time for isolated vehicles. The vehicle density has no effect on the re-healing time, as the scenario is conditioned to isolated vehicles - therefore, we plot this delay against the RSU density, for densities between 0.5 and 1.25 units $/ \mathrm{km}$.

Observer that there is an excellent match between the simulation results and the results of our analysis. Re-healing time is seen to increase linearly with the increase in distance between RSUs, which is an expected result given the fact that vehicles travel at a fixed speed.

The second scenario with connected RSUs considers that the target vehicle is part of a cluster. The length of the cluster the vehicle is part of depends directly on the vehicle density, higher densities leading to larger clusters.

Figure $4 d$ plots the average re-healing time of vehicles that are part of clusters, for a fixed RSU spacing of $1 \mathrm{~km}$. Again, we observe a very good match between simulation results and our analytical models. Comparing with the $1000 \mathrm{~m}$ data point in Figure $4 \mathrm{c}$, which corresponds to $\approx 9$ seconds, one can conclude that being part of a cluster considerably improves the vehicle's time to connect to an infrastructure.

The excellent match between models and simulation results in Figures (4a), (4b), (4c), and (4d), for both special cases and global end-to-end delays, uphold the validity of our analytical models and the conviction that all relevant possibilities for interactions with RSUs have been considered.

\section{Single-Gap Communication Delay}

We consider a road where RSUs are deployed in intervals of 1000 and 750 meters, and, conservatively, have the same 


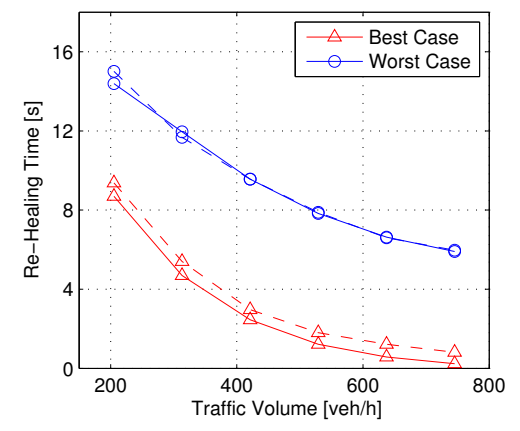

(a)

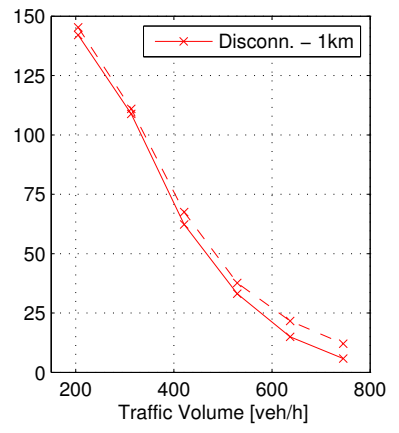

(b)

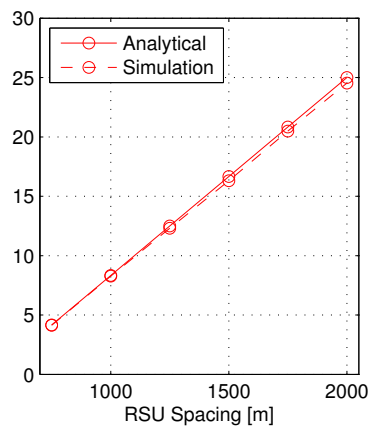

(c)

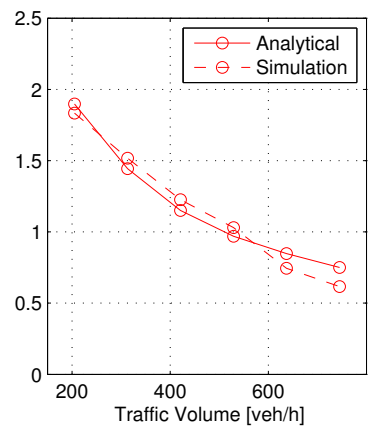

(d)

Figure 4. (a) Best-case and worst-case re-healing times with disconnected RSUs at $1 \mathrm{~km}$ intervals; (b) Total re-healing time for vehicles spaced $10 \mathrm{~km}$ apart, with disconnected RSUs; (c) re-healing time for an isolated vehicle with connected RSUs; (d) re-healing time for a clustered vehicle with connected RSUs.

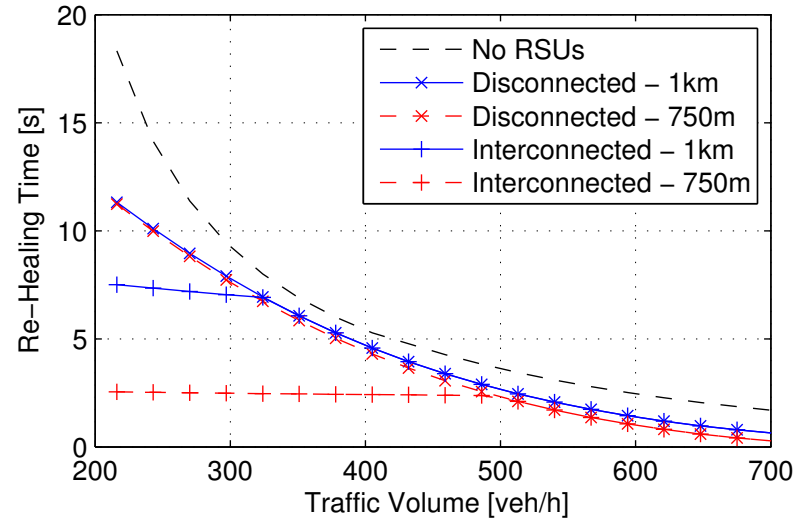

Figure 5. Single-gap re-healing time.

radio range as vehicles. Vehicle speeds were set to $30 \mathrm{~m} / \mathrm{s}$ (both lanes), and radio ranges to $250 \mathrm{~m}$. Figure 5 shows a comparison of all three scenarios, for densities that go from $\approx 200$ to $\approx 700$ cars per hour. Since it has been shown that the analytical models are in line with the simulation results, all plots for the remainder of this section feature data from the models only.

It can be observed that a deployment of isolated RSUs can yield a reduction of 1 to 5 seconds in the network's mean re-healing time. This advantage becomes smaller for denser networks, as disconnection becomes less of an issue.

A deployment of connected RSUs shows substantial improvement for very sparse networks, for densities under $\lambda \approx 325 \mathrm{veh} / \mathrm{h}$ for $C_{I}=1000 \mathrm{~m}$, and $\lambda \approx 475 \mathrm{veh} / \mathrm{h}$ for $C_{I}=750 \mathrm{~m}$. Due to the way the scenario for connected RSUs was designed, note that, for a transmission over a single hop and given sufficient vehicle density in both lanes, opposite lane vehicles may be able to deliver a message in shorter time than if going through connected RSUs. This is evident from Figure 5, for higher densities.

For a single-hop transmission, we observe that the best rehealing time in a road with connected RSUs is the shortest time between the opposite-lane store-carry-forward approach, and the delivery-through-infrastructure approach. This is particularly valid for safety messages, where an ideal vehicular

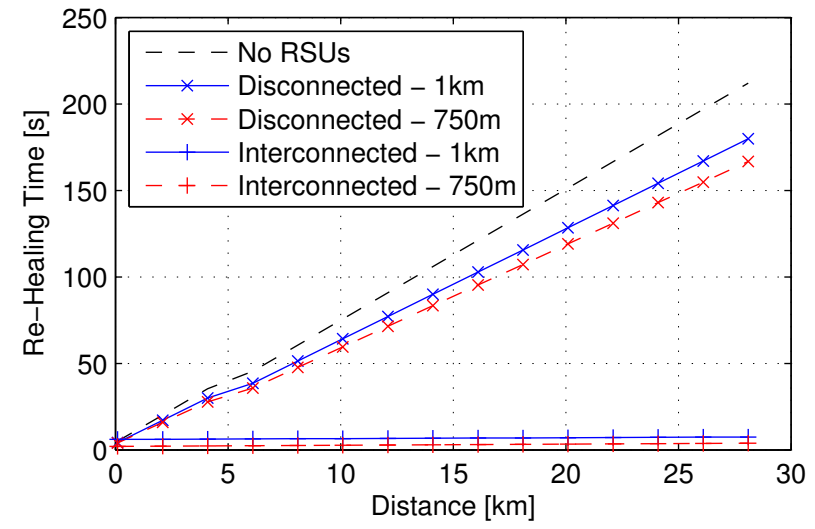

Figure 6. Total end-to-end delay over large distances.

network must try to broadcast the message through any possible means, and the re-healing metric is the shortest time for that message to be delivered.

\section{End-To-End Communication Delay}

In a multi-gap scenario, we study the delay involved in sending a message over a large segment of road (up to $30 \mathrm{~km}$ ), which is essentially an accumulation of multiple re-healing delays, based on the number of clusters between source and destination, and on the inter-cluster spacing.

In the no-RSU and disconnected RSU models, store-carryforward is the primary mechanism of transmission. For these scenarios, we fix the vehicle density in both directions and determine the mean number of clusters to be traversed. Then, the total delay is the sum of all re-healing times required to get a message from each cluster to the next. For connected RSUs, we determine the number of RSUs the message must travel to reach the destination, and add a conservative $50 \mathrm{~ms}$ delay per hop to the re-healing time.

Figure 6 plots accumulated re-healing times for all three scenarios as a function of the length of road to transmit a message across, in a network where $\lambda$ is fixed to a value indicative of a sparse network $(\lambda=425 \mathrm{veh} / \mathrm{h})$. All other parameters are the same as in the single-gap scenario above. 
This is a scenario where a deployment of RSUs is capable of yielding significant gains. The presence of disconnected RSUs steadily reduces the multi-gap accumulated delay by $\approx 20 \%$, with higher gains possible for closer-spaced RSUs. The gains, however, are not what one would hope for. In this particular scenario, when RSUs are deployed $1 \mathrm{~km}$ apart with a $250 \mathrm{~m}$ range, $50 \%$ of the road becomes covered by the RSUs alone, yet the re-healing time gains are modest at best.

Interconnected RSUs, on the other hand, as expected, show significant gains. A deployment of interconnected RSUs can thus be viewed as the only way to carry messages across long lengths of road with delays not exceeding 5 seconds. The results depicted in Figure 6 are the key finding of the paper: the deployment of RSUs can only bring significant improvements if they are connected. In other words, deploying a high number of disconnected RSUs will not lead to significant benefits.

We have also studied the probability distributions of the rehealing time of connected and and disconnected RSUs, and found that the fluctuations around the statistical average are very small.

\section{EXPERIMENTAL VERIFICATION}

To further strengthen the validity of the proposed models for RSU-aided vehicular communication, we designed and implemented a physical testbed for experimental verification of VANET technologies in Aveiro, Portugal. Our testbed comprises five hardware units, each consisting of a modern Single Board Computer (SBC) fitted with an IEEE $802.11 \mathrm{p}$ radio enhanced to operate in the licensed 5.9 GHz ITS band [22].

\section{A. Testbed Hardware and Software}

- SBC: PCEngines Alix3D3, with a $500 \mathrm{MHz}$ AMD Geode LX800 CPU at its core (32-bit x86 architecture) [27].

- Radio: Unex DCMA-86P2 5.86-5.92 GHz miniPCI radio for $802.11 \mathrm{p} / \mathrm{DSRC}$ applications (Atheros AR5414A-B2B chipset) [28].

- Antenna: L-Com 5.1-5.9 GHz $5 \mathrm{dBi}$ omnidirectional antenna.

- Operating System: Linux Debian 'Squeeze', kernel version 2.6.32-5-486.

- Driver: Modified ath5k based on version 'compatwireless-2010-11-01'.

At the time of this writing, the publicly-available drivers for Atheros chipsets supported neither the $802.11 \mathrm{p}$ standard nor any hardware operating at $5.9 \mathrm{GHz}$. For this reason, we have modified the 'ath5k' driver that is freely available for Atherosbased chipsets, implemented the set of $802.11 \mathrm{p}$ standards, and modified the Central Regulatory Domain Agent (CRDA) and the software's regulatory database to allow operation in the $5.9 \mathrm{GHz}$ band.

For the mobile units, we installed hardware in a range of common mid-size vehicles, deploying the units on the rooftops of the vehicles. For the fixed RSUs, we installed battery-powered hardware in tripods that were then deployed on the side of highways, at the same height as mobile units. To connect RSUs for the interconnected RSU tests, we used 802.11g hardware operating in the $2.4 \mathrm{GHz}$ ISM band.

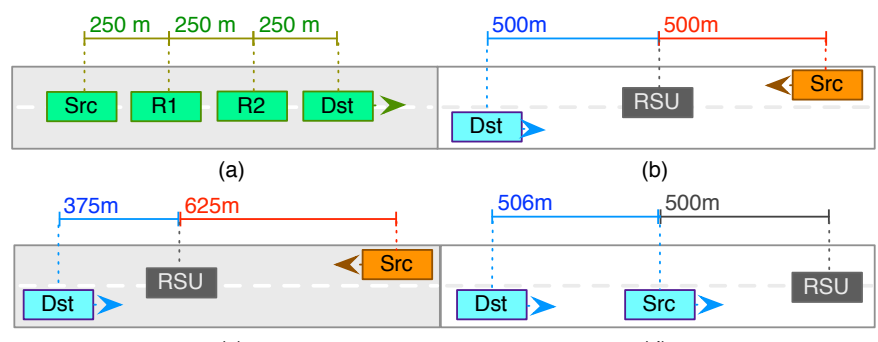

(c)

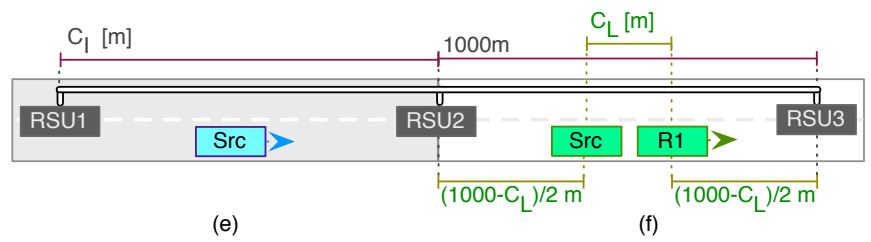

Figure 7. (a) Experimental setup for multi-hop communication; (b), (c), (d) disconnected RSU setups, scenarios D1, D2, and D3, respectively; (e), (f) connected RSU setups, isolated vehicle and clustered vehicles, respectively.

\section{B. Case Scenarios and Setup}

To evaluate the validity of our models, a number of experimental measurements were carried out to evaluate the performance of RSU-supported sparse highway networks. These include the verification of real-life radio range of $802.11 \mathrm{p}$ radios, multi-hop communication delay, and backbone interconnection of RSUs.

All tests were performed in a low-traffic, $2 \mathrm{~km}$ straight road at the edge of the city of Aveiro, Portugal. For safety reasons and to comply with local road regulations, the drivers maintained the mobile units at a fixed $50 \mathrm{~km} / \mathrm{h}$ speed, and therefore all the analytical data points to which our experimental data is matched with are derived for a speed of $13.89 \mathrm{~m} / \mathrm{s}$. Regarding the radio's transmission range, after calibration all our radios exhibited $0 \%$ packet loss at $240 \mathrm{~m}$ range, which then decayed to having no communication at distances of 260-270 meters, which matches well with our theoretical $250 \mathrm{~m}$ radio range. For this behavior, the transmission power measured at the radio's output was $4 \mathrm{dBm}$.

To begin with, the assumption that multi-hop communication has sufficiently low delay to be considered negligible was verified. The first scenario, depicted in Figure 7a, places four vehicles in a row, each at a $250 \mathrm{~m}$ radio range to match the maximum radio range that we assume throughout this work, and attempts a 3-hop communication. The results of this test, given in Table I, show that the delays are at least two orders of magnitude lower than the re-healing times given in this paper, and can therefore be safely ignored.

The exhaustive evaluation of disconnected RSU scenarios is a complex and cumbersome process, due to the number of possible sub-scenarios that the model deals with. For disconnected RSUs, three sample scenarios that represent the

\begin{tabular}{c|c|c}
\hline One hop & Two hops & Three hops \\
\hline $0.49 \mathrm{~ms}$ & $5.51 \mathrm{~ms}$ & $15.58 \mathrm{~ms}$ \\
\hline
\end{tabular}

Table I

EXPERIMENTAL MULTI-HOP COMMUNICATION DELAYS. 
main communication systems in such scenarios were designed, and are depicted in Figures $7 \mathrm{~b}, 7 \mathrm{c}$ and $7 \mathrm{~d}$.

- Scenario D1: in this scenario, a $S r c$ and a Dst vehicle are positioned at equal relative distance to an RSU; in such a setup, the RSU should provide maximum benefit and reduce the distance the vehicles must travel for a message to be delivered to Dst.

- Scenario D2: similar to Scenario D1, but in this case we position the RSU in a location where it is at the edge of providing no benefit to the communication. The expectation is for the transmission to proceed as if no RSU was present.

- Scenario D3: in this scenario, both $S r c$ and Dst travel east-bound on the same lane, but are disconnected. An RSU is placed away from both vehicles, and is expected to receive Src's message, hold it, and deliver it to Dst.

For scenarios where connected RSUs are deployed, the experimental setup is easier to automate, and so we directly verified the analytical and simulation results previously given in Figures $4 \mathrm{c}$ (isolated vehicle) and $4 \mathrm{~d}$ (clustered vehicle), now with a speed of $13.89 \mathrm{~m} / \mathrm{s}$ as reference. Figures $7 \mathrm{e}$ and $7 \mathrm{f}$ show the two setups. In both cases, the backbone between the two RSUs was established with $802.11 \mathrm{~g}$ radios operating in the $2.4 \mathrm{GHz}$ ISM band.

For isolated vehicles (Figure 7e), a single car, Src, was designed to generate a new message as soon as it reached the middle of two RSUs, and measured the delay for that message to reach the opposite-end RSU1 (which requires backbone relaying of the message from RSU2). The tests were then repeated for different separation distances between the RSUs.

For clustered vehicles (Figure 7f), the setup is similar but in this case, instead of a single car, one has a cluster formed by three vehicles. The tail of the cluster generates a message as soon as the cluster is in the middle of two RSUs which are $1 \mathrm{~km}$ apart, and the time for that message to reach RSU2 is measured. The tests were then repeated for different cluster lengths, according to the mean cluster lengths expected for a road with vehicle densities between 95 and $345 \mathrm{veh} / \mathrm{h}$.

\section{Experimental Results and Discussion}

Figure 8a plots the analytical delay predictions and experimental results for scenarios D1, D2 and D3. The results show, for the three test scenarios under consideration, that the basic communication in disconnected RSU-supported networks can be expected to operate as per the assumptions upon which our analytical models are built, and that analytical results closely match the experimental data.

The experimental results for the scenarios with interconnected RSUs, for isolated and cluster vehicles respectively, can be seen in Figures $8 \mathrm{~b}$ and 8c. Once again, the experimental results closely follow our analytical model's predictions, both for isolated and clustered vehicles, and both for various RSU spacings and various traffic densities, further strengthening the validity of these models.

These are very important empirical results as they show how close the analytical models follow the reality of vehicular communications. The close match between our analytical models and experimental results with real-life $802.11 \mathrm{p}$ equipment are a key result.

\section{DISCUSSION}

Our results show that RSUs are indeed able to significantly decrease the re-healing time in sparse vehicular networks if the deployed RSUs are interconnected. How to interconnect the RSUs in real-life is an important practical issue - many highways now carry a fiber optic backbone to which RSUs can ideally be attached to, or are covered by broadband cellular wireless access, though the latter has a much lower bandwidth when compared to the fiber optic backbone. Regardless, these solutions require leasing the existing infrastructure to a thirdparty, which would incur additional costs.

New wireless technologies may also be viable for establishing point-to-point links between RSUs. In particular, upcoming technologies such as WiMAX and LTE have been shown capable of establishing point-to-point links over tens of kilometers (whereas we expect RSUs to be deployed at much closer distances) while providing more throughput than the 802.11p theoretical maximum [29], [30].

An important observation of this study is that disconnected RSUs, when deployed with standard equipment, provide modest improvements to the network's re-healing time, whereas the interconnection of RSUs results in tremendous improvement, as shown in Figure 6.

Regarding possible application scenarios, our analytical models can be used to show how an infrastructure-assisted vehicular network can be designed in order to support protocols with specific delay requirements. For example, to prepare a deployment to support a routing protocol such as AODV (which requires a maximum per-gap delay of 2 seconds [31]) when $\lambda=425[\mathrm{veh} / \mathrm{h}]$, one would need to deploy disconnected RSUs every $295 \mathrm{~m}$, or connected RSUs every $740 \mathrm{~m}$.

Finally, highway entries and exits should be taken into account in any comprehensive analysis. The main consequence of such structures is a change in vehicle density. By splitting the highway into shorter segments of road, at entries and exits, each segment can be analyzed independently, computing each mean re-healing time, and aggregating the results.

\section{RELATED WORK}

The dynamics of two-way highway traffic and VANET capacity have been studied in previous works such as [1][4], with detailed analysis on the network performance and information storage capability of the VANET. The physical distribution of RSUs to optimize communication is an important design consideration for real-life scenarios, and studies exist on the placement of RSUs for Internet access [5], [6], RSU deployment at popular junctions [7], and determination of critical network points [8]-[10]. All these works are based on urban areas. Network connectivity in V2I has also been an important research area [11]-[13], where the tradeoffs between RSU density/placement and overall VANET connectivity can be determined.

Information dissemination and Internet access are the subject of many works [14]-[16], all of which use a V2I approach, relegating $\mathrm{V} 2 \mathrm{~V}$ communications between vehicles to 


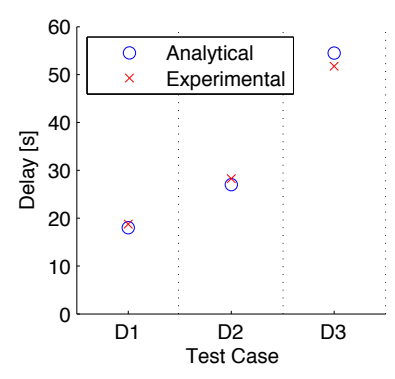

(a)

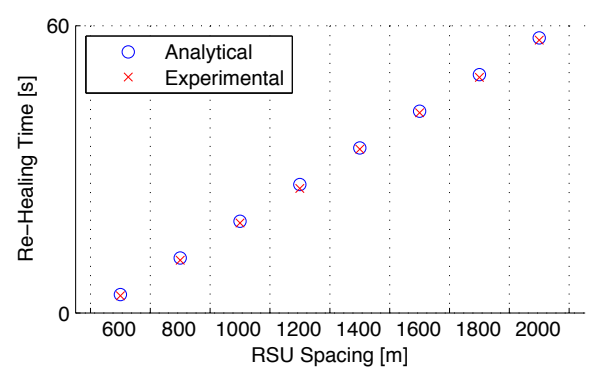

(b)

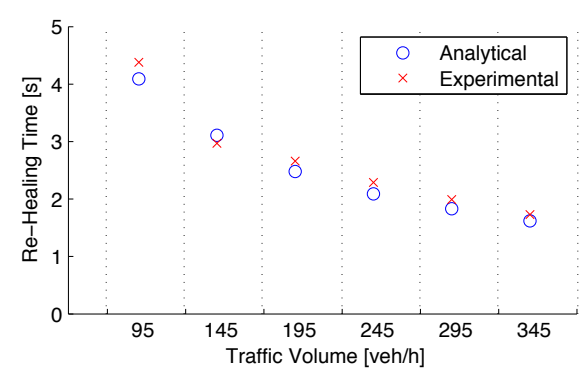

(c)

Figure 8. Experimental results for: (a) test scenarios D1, D2, and D3, of disconnected RSUs; (b) an isolated vehicle with interconnected RSUs; (c) a clustered vehicle with interconnected RSUs.

a secondary role. Others study the RSU network's ability to deliver messages over large distances [17], [18], but consider uni-directional traffic and disconnected infrastructure only.

Of more relevance to this work are studies [19] and [20], which also approach the relation between infrastructure density and network delay performance. The former, [19], considers both two-way traffic and the same store-carry-forward method for infrastructure-less communication, but with a different viewpoint for the concept of re-healing: the authors consider that re-healing is achieved once the message is relayed to an RSU or to a vehicle in the region of interest. The latter, [20], studies vehicle-to-RSU packet delivery delay and RSU separation distance, and obtains a maximum delay violation bound. The study does not, however, consider packet retransmission via opposite-lane vehicles, which is an important method for message forwarding in highways.

It is important to mention that among these studies, very few address bidirectional communication, and none of them gives a clear comparison between using connected and disconnected RSUs in quantitative terms. Moreover, none of the aforementioned studies is complemented with real-life testbed results. The work reported in this paper aims to bridge these gaps.

\section{CONCLUSIONS}

It has been shown that, contrary to conventional wisdom, deployments of RSUs on highways provide little or no improvement in the re-healing time or the end-to-end delay in safety message dissemination if the RSUs are disconnected from each other. Conversely, interconnected RSUs can reduce the re-healing time or the end-to-end delay by orders of magnitude. In countries where broadband wired or wireless communications capability exists along major highways, this does not pose any serious problems. When such capability does not exist, however, the results of this work have shown that for significant improvements the RSUs must be connected. The analysis, the simulations, and the experiments conducted all endorse the validity of these observations and conclusions. We believe that these findings bring a new perspective to how the RSU deployment issue should be handled.

\section{REFERENCES}

[1] N. Wisitpongphan, Fan Bai, P. Mudalige, V. Sadekar, and O.K. Tonguz, "Routing in Sparse Vehicular Ad Hoc Wireless Networks," IEEE Journal on Selected Areas in Communications, 2007, vol. 25, no. 8.

[2] Bojin Liu, B. Khorashadi, D. Ghosal, Chen-Nee Chuah, and M.H. Zhang, "Assessing the VANET's Local Information Storage Capability under Different Traffic Mobility," 2010 Proceedings IEEE INFOCOM.

[3] A. Zemlianov and G. de Veciana, "Capacity of ad hoc wireless networks with infrastructure support," IEEE Journal on Selected Areas in Communications, vol. 23, no. 3, pp. 657-667, Mar. 2005,

[4] M. Nekoui, A. Eslami, and H. Pishro-Nik, "The capacity of Vehicular Ad Hoc Networks with infrastructure," WiOPT 2008: 6th International Symposium on Modeling and Optimization in Mobile, Ad Hoc, and Wireless Networks and Workshops, pp. 267-272, Apr. 2008.

[5] Pan Li, Xiaoxia Huang, Yuguang Fang, and Phone Lin, "Optimal Placement of Gateways in Vehicular Networks," IEEE Transactions on Vehicular Technology, vol. 56, no. 6, pp. 3421-3430, Nov. 2007.

[6] K. Mershad and H. Artail, "Using RSUs as Delegates for Pervasive Access to Services in Vehicle Ad Hoc Networks," 17th IEEE International Conference on Telecommunications, Doha, Qatar, April, 2010.

[7] A. Kchiche and F. Kamoun, "Centrality-based Access-Points deployment for vehicular networks," IEEE 17th International Conference on Telecommunications, 2010, pp. 700-706.

[8] C. Lochert, B. Scheuermann, C. Wewetzer, A. Luebke, and M. Mauve, "Data Aggregation and Roadside Unit Placement for a VANET Traffic Information System," Proc. of the 5th ACM Int'l Workshop on Vehicular Internetworking, San Francisco, USA, Sep. 2008, pp. 58-65.

[9] S. Busanelli, G. Ferrari, V.A. Giorgio, and N. Iotti, "Comparative investigation of single-hop and multi-hop broadcast strategies for information dissemination in VANETs," 2011 11th International Conference on ITS Telecommunications (ITST), pp. 738-743, Aug. 2011.

[10] Seh Chun Ng, Wuxiong Zhang, Yu Zhang, Yang Yang, and Guoqiang Mao, "Analysis of Access and Connectivity Probabilities in Infrastructure-Based Vehicular Relay Networks," 2010 IEEE Wireless Communications and Networking Conference (WCNC), Apr. 2010.

[11] Wuxiong Zhang, Yu Chen, Yang Yang, Xiangyang Wang, Yu Zhang, Xuemin Hong, and Guoqiang Mao, "Multi-Hop Connectivity Probability in Infrastructure-Based Vehicular Networks," IEEE Journal on Selected Areas in Communications, 2012, vol. 30, no. 4.

[12] Yang Yang, Zhenqiang Mi, J.Y. Yang, and Guangjun Liu, "A Model Based Connectivity Improvement Strategy for Vehicular Ad hoc Networks," 2010 IEEE 72nd Vehicular Technology Conference Fall (VTC 2010-Fall), pp. 1-5, Sep. 2010.

[13] S. Yousefi, E. Altman, R. Elazouzi, and M. Fathy, "Improving connectivity in vehicular ad-hoc networks: An analytical study," Computer Communications, vol. 31, 2008, pp. 1653-1659.

[14] B. Aslam, and C.C. Zou, "Optimal roadside units placement along highways," 2011 IEEE Consumer Communications and Networking Conference (CCNC), pp. 814-815, Jan. 2011.

[15] C. Lochert, B. Scheuermann, M. Caliskan, and M. Mauve, "The feasibility of information dissemination in vehicular ad-hoc networks," 4th Annual Conference on Wireless on Demand Network Systems and Services, January 2007, pp. 92-99.

[16] P. Salvo, F. Cuomo, A. Baiocchi, and A. Bragagnini, "Road Side Unit coverage extension for data dissemination in VANETs," 2012 9th Annual Conference on Wireless On-demand Network Systems and Services (WONS), pp. 47-50, Jan. 2012. 
[17] K. Mershad, H. Artail, and M. Gerla, "We Can Deliver Messages to Far Vehicles," IEEE Transactions on Intelligent Transportation Systems.

[18] A. Abdrabou and W. Zhuang, "On a Stochastic Delay Bound for Disrupted Vehicle-to-Infrastructure Communication with Random Traffic," IEEE Global Telecommunications Conference, 2009, pp. 1-6.

[19] Sok-Ian Sou and O.K. Tonguz, "Enhancing VANET Connectivity Through Roadside Units on Highways," IEEE Transactions on Vehicular Technology, vol. 60, no. 8, pp. 3586-3602, Oct. 2011.

[20] A. Abdrabou and Weihua Zhuang, "Probabilistic Delay Control and Road Side Unit Placement for Vehicular Ad Hoc Networks with Disrupted Connectivity," IEEE Journal on Selected Areas in Communications, vol. 29, no. 1, pp. 129-139, Jan. 2011.

[21] A.B. Reis, S. Sargento, and O.K. Tonguz, "On the Performance of Sparse Vehicular Networks with Road Side Units," 2011 IEEE 73rd Vehicular Technology Conference, 2011.

[22] A. Cardote, F. Neves, S. Sargento, and P. Steenkiste, "A statistical channel model for realistic simulation in VANET," 2012 IEEE Vehicular Networking Conference (VNC), Nov. 2012, pp. 48-55.

[23] California Center for Innovative Transportation, "Berkeley Highway Lab (BHL)," n.d. [Online]. Available: http://bhl.calccit.org/

[24] John R. McLean, "Two-Lane Highway Traffic Operations - Theory and Practice," Gordon and Breach Science Publishers, 1989.

[25] L. Kleinrock, "Queuing Systems," Wiley Interscience, p. 45, 1975.

[26] A.B. Reis, "Numerical Computation of Cluster Length PDF," n.d. [Online]. Available: http://www.ece.cmu.edu/ abragare/vehicular/ .

[27] PC Engines GmbH, "PC Engines alix3d3 product file," Oct 26, 2010. [Online]. Available: http://pcengines.ch/alix3d3.htm

[28] Unex Technology Corp., "DCMA-86P2: industrial grade, high power $5.865 .92 \mathrm{GHz}$ wifi mini-PCI module for $802.11 \mathrm{p} / \mathrm{DSRC}$ application, AR5414A-B2B," n.d. [Online]. Available: http://www.unex.com. tw/product/dcma-86p2 .

[29] L. Nuaymi, "WiMAX: Technology for Broadband Wireless Access". West Sussex, England: John Wiley \& Sons, 2007.

[30] S. Sesia, M. Baker, and I. Toufik, "LTE: The UMTS Long Term Evolution: from Theory to Practice". John Wiley \& Sons, 2. ed., 2011.

[31] C. Perkins et al., "Ad hoc On-Demand Distance Vector (AODV) Routing," IETF RFC 3561, July 2003.

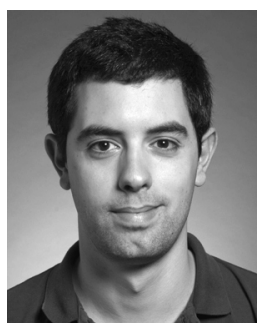

Andre B. Reis is a Ph.D. candidate in Electrical and Computer Engineering with Carnegie Mellon University and University of Aveiro, under the CMUPortugal Program. His current research is focused on infrastructure support systems for vehicular networks in challenging scenarios. He has also published on WiMAX and multimedia Quality of Experience on ad hoc networks. Andre received his B.S. and M.Sc. in Electronics and Telecommunications Engineering from University of Aveiro, in 2009, in collaboration with the Eindhoven University of Technology (TU/e, Netherlands) and Karlstad University (Sweden).

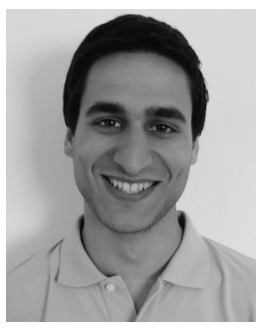

Filipe Neves studied Electronic and Telecommunications Engineering at University of Aveiro, Portugal, and received his M.Sc. degree in 2011. Between 2011 and 2012, he was a Researcher Grant Holder at Institute of Telecommunications - Aveiro, working on DRIVE-IN project (a CMU-Portugal research project). He has published 3 papers in the area of wireless networking and he works on ad-hoc networks, especially in vehicular networks. Recently, Filipe joined the Veniam 'Works team.

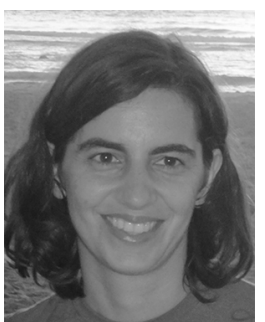

Susana Sargento (http://www.av.it.pt/ssargento) received her Ph.D. in 2003 in Electrical Engineering. She joined the Department of Computer Science of the University of Porto in September 2002, and is in the Universidade de Aveiro and the Instituto de Telecomunicacoes since February 2004, where she is leading the Network Architectures and Protocols (NAP) group (http://nap.av.it.pt). She is also a Guest Faculty of the Department of Electrical and Computer Engineering from Carnegie Mellon University, USA, since August 2008, where she performed Faculty Exchange in 2010/2011. She has been involved in several national and European projects, taking leaderships of several activities in the projects, such as the QoS and ad-hoc networks integration activity in the FP6 IST-Daidalos Project. She has been recently involved in several FP7 projects (4WARD, Euro-NF, C-Cast, WIP, Daidalos, C-Mobile), national projects, and Carnegie Mellon Portugal research projects (DRIVE-IN with the Carnegie Melon University). She has been TPC-Chair and organizing several conferences, such as MONAMI'11, NGI'09, IEEE ISCC'07, NTMS'12, IEEE FEDNET (with IEEE NOMS'12) and ISCC 2013. She has also been a reviewer of numerous international conferences and journals, such as IEEE Wireless Communications, IEEE Networks, IEEE Communications. Her main research interests are in the areas of Next Generation and Future Networks, more specifically QoS, mobility, self- and cognitive networks. She regularly acts as an Expert for European Research Programmes.

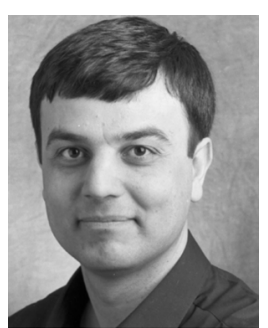

Ozan K. Tonguz is a tenured Full Professor in the Department of Electrical and Computer Engineering at Carnegie Mellon University (CMU). Before joining CMU in August 2000, he was with the ECE Dept. of the State University of New York at Buffalo (SUNY/Buffalo). He joined SUNY/Buffalo in 1990 as an Assistant Professor, where he was granted early tenure and promoted to Associate Professor in 1995, and to Full Professor in 1998. Prior to joining academia, he was with Bell Communications Research (Bellcore) between 1988-1990 doing research in optical networks and communication systems. His current research interests are in vehicular networks, sensor networks, computer networks, wireless networks and communications systems, biologically inspired self-organizing networks, smart grid, optical communications and networks, bioinformatics, and security. He has published close to 300 technical papers in IEEE journals and conference proceedings. He is well-known for his contributions in vehicular networks, wireless communications and networks, and optical communications and networks. His work on iCAR (the Integrated Cellular and Ad Hoc Relay Systems) is internationally acclaimed as well. He is the author (with G. Ferrari) of the Wiley book entitled "Ad Hoc Wireless Networks: A Communication-Theoretic Perspective". He was the architect of the "High Performance Waveform (HPW)" that was implemented in Harris RF Communications' AN/PRC-117f UHF band man-pack tactical radio. He is the founder and President of Virtual Traffic Lights, LLC, a CMU start-up which was launched in December 2010 for providing solutions to several key transportation problems related to safety and traffic information systems, using vehicle-to-vehicle and vehicle-to-infrastructure communications paradigms. His industrial experience includes periods with Bell Communications Research, CTI Inc., Harris RF Communications, Aria Wireless Systems, Clearwire Technologies, Nokia Networks, Nokia Research Center, Neuro Kinetics, Asea Brown Boveri (ABB), General Motors (GM), Texas Instruments, and Intel. He currently serves or has served as a consultant or expert for several companies, major law firms (Jones Day, WilmerHale, Williams and Connolly, Heller Ehrman, Baker Botts, Soroker-Agmon, Dinsmore\&Shohl, Carlson and Caspers, etc.), and government agencies (such as NSF) in the USA, Europe, and Asia in the broad area of telecommunications and networking. He also served as the Co-Director (Thrust Leader) of the Center for Wireless and Broadband Networking Research at Carnegie Mellon University. In addition to serving on the Technical Program Committees of several IEEE conferences, he has served as an Associate Editor of IEEE Transactions on Communications, IEEE/OSA Journal of Lightwave Technology, IEEE Communications Magazine, and Elsevier Ad Hoc Networks Journal.

More details about his research interests, research group, projects, and publications can be found at http://www.ece.cmu.edu/ tonguz/. 